\title{
STAR CENTER POINTS OF STARLIKE FUNCTIONS
}

\author{
LOUIS RAYMON and DAVID E. TEPPER*
}

(Received 3 July 1973; revised 12 December 1973)

Communicated by E. Strzelecki

\section{Introduction}

Let

$$
w=f(z)=z+\sum_{n=2}^{\infty} a_{n} z^{n}
$$

be regular and univalent in the unit disk $D$ and map $D$ onto a region $R$. A point $w_{0} \in R$ is called a star-center point of $f(z)$, or of $R$, if $t f(z)+(1-t) w_{0} \in R$ for $z \in D$ and $0 \leqq t \leqq 1$. In this paper we consider only functions of the form (1) where $w=.0$ is a star-center point, i.e., those functions that are starlike with respect to the origin.

Given $w=f(z)$ as in (1), we define the index of starlikeness of $w=f(z)$ to be

$$
\delta=\sup \{r \mid f(z) \text { is a star-center point of } f(D) \text { whenever }|z| \leqq r\} .
$$

We denote by $S_{\delta}$ the class of all starlike functions whose index is equal to $\delta$, $0 \leqq \delta \leqq 1$.

From the definition it follows that $S_{0}$ and $S_{1}$ are the classes of normalized starlike and convex univalent functions respectively. In this note we obtain estimates for $\left|a_{n}\right|,|f(x)|$ and $\operatorname{Re}\left[z f^{\prime}(z) / f(z)\right]$ when $w=f(z) \in S_{\delta}$.

NotATiOn: Let $D_{r}$ denote the disk $|z|<r$. Let $\phi(z, a, \alpha)=e^{i \alpha}(z-a)(1-\bar{a} z)^{-1}$, where $-\pi<\alpha \leqq \pi$ and $|a|<1$.

\section{Preliminaries}

In this section we give some necessary and sufficient conditions for a function to belong to the class $S_{\delta}$.

\footnotetext{
* Supported in part by a National Science Foundation Grant.
} 
Lemma 1. Let $w=f(z)$ be of the form (1). Then $w=f(z) \in S_{\delta}$ if and only if the function

$$
F(z)=t f(z)+(1-t) f(\delta z \phi(z, a, \alpha))
$$

is subordinate to $w=f(z)$ in $D$ for $-\pi<\alpha \leqq \pi,|a|<1,0 \leqq t \leqq 1$.

PRoOF. If $w=f(z) \in S_{\delta}$, then the subordination holds because $|\phi(z, a, \alpha)|<1$.

Suppose the subordination holds. For $\delta=0$ the result is well-known, so we may suppose $\delta>0$. Given $z_{0},\left|z_{0}\right|<\delta$, we show $f\left(z_{0}\right)$ is a star-center point of $f\left(D_{r}\right)$ for $r>\delta^{-1}\left|z_{0}\right|$. For each $z,|z|>\delta^{-1}\left|z_{0}\right|$, let

$$
A=\delta^{-1} \bar{z}^{-1} \bar{z}_{0}, \alpha=\operatorname{Arg}(1-\bar{A} \bar{z})(1-A z)^{-1}, a=-(\bar{A}-z)(1-\bar{A} \bar{z})^{-1} .
$$

Then $z_{0}=\delta z \phi(z, a, \alpha)$ and (3) yields $t f(z)+(1-t) f\left(z_{0}\right)=f(\zeta)$ for some $\zeta$ in $D$. By well-known properties of subordination, $|\zeta|<|z|$, (see [1, p. 227]).

LEMMA 2. The function $w=f(z) \in S_{\delta}, \delta>0$, if and only if

$$
\operatorname{Re} \frac{z f^{\prime}(z)}{f(z)-f(\zeta)}>0
$$

for $|\zeta|<\delta,|\zeta|<|z|<1$.

Proof. Choose $\varepsilon>0$ so that $|\zeta|+\varepsilon<\delta$, and $r>0$ so that $|\zeta| \delta^{-1}<r<1$. Since $f\left(D_{\delta}\right)$ is a convex set, (5) holds for $|z|=|\zeta|+\varepsilon$. By Lemma 1, (5) also holds for $|z|=r$. Using the, minimum modulus principle for harmonic functions (5) follows upon letting $\varepsilon \rightarrow 0$ and $r \rightarrow 1$.

Lemma 3. The function $w=f(z), \varepsilon S_{\delta}$ if and only if

$$
G(z, \phi)=\frac{z f^{\prime}(z)}{f(z)-f(\delta z \phi(z, a, \alpha))}
$$

has positive real part for $|z|<1,|a|<1,-\pi<\alpha \leqq \pi$.

Proof. For $\delta=0$ the result is well-known. If $\delta>0$, then given $z_{0},\left|z_{0}\right|<\delta$ and $z,|z|>\delta^{-1}\left|z_{0}\right|$, we use (4) to find a function $\phi(z, a, \alpha)$ that satisfies the equation $\delta z \phi(z, a, \alpha)=z_{0}$. For $\zeta=z_{0}$, (6) follows from (5).

\section{Distortion theorems}

THEOREM 1. If $w=f(z) \in S_{\delta}, 0 \leqq \delta \leqq 1$, then

$$
\frac{1-|z|}{(1-\delta|z|)(1+|z|)} \leqq \operatorname{Re} \frac{z f^{\prime}(z)}{f(z)} \leqq \frac{1+|z|}{(1+\delta|z|)(1-|z|)},
$$

equality holding in the cases $\delta=0$ and $\delta=1$. 
Proof. By a well-known theorem (Nehari (1952); page 173),

$$
[\operatorname{Re} G(0, \phi)] \frac{1-|z|}{1+|z|} \leqq \operatorname{Re} G(z, \phi) \leqq[\operatorname{Re} G(0, \phi)] \frac{1+|z|}{1-|z|},
$$

which becomes

$$
\begin{aligned}
{\left[\operatorname{Re} \frac{1}{1+e^{i \alpha} a \delta}\right] \frac{1-|z|}{1+|z|} } & \leqq \operatorname{Re} \frac{z f^{\prime}(z)}{f(z)-f(\delta z \phi(z, a, \alpha))} \\
& \leqq\left[\operatorname{Re} \frac{1}{1+e^{i \alpha} a \delta}\right] \frac{1+|z|}{1-|z|} .
\end{aligned}
$$

Letting $\alpha=-\arg a+\pi$ and $z=a$ on the left side of (9) we obtain

$$
\frac{1}{1-\delta|a|} \frac{1-|a|}{1+|a|} \leqq \operatorname{Re} \frac{a f^{\prime}(a)}{f(a)},
$$

which is equivalent to the left side of (7). The right hand side of (7) is obtained similarly.

It is interesting to note that for $\delta=1$, we obtain the well-known result that $\operatorname{Re}\left[z f^{\prime}(z) / f(z)\right] \geqq(1+|z|)^{-1}$; see (Strohäcker (1933)) or more recently (Suffridge (1940));

THEOREM 2. If $w=f(z) \in S_{\delta}$, then

$$
|z| \frac{(1-\delta|z|)^{(1-\delta) /(1+\delta)}}{(1+|z|)^{2 / 1+\delta}} \leqq|f(z)| \leqq|z| \frac{(1+\delta|z|)^{(1-\delta) /(1+\delta)}}{\left(1-|z|^{2 / 1+\delta}\right.}
$$

equality holding in the cases $\delta=0$ and $\delta=1$.

Proof. Using the identity

$$
\frac{\partial}{\partial|z|} \log \left|\frac{f(z)}{z}\right|=\frac{1}{|z|}\left|\operatorname{Re} \frac{z f^{\prime}(z)}{f(z)}-1\right|,
$$

(10) is obtained upon integrating (7).

\section{Coefficient estimates}

We wish to give coefficient estimates for the expansion (1) when $w=f(z) \in S_{\delta}$. If $w=f(z) \in S_{\delta}$, then

$$
\operatorname{Re} \frac{z f^{\prime}(z)}{f(z)-f(-\delta z)}>0
$$

for $z \in D$. This is so because (5) holds for all $\zeta,|\zeta|<\delta$. If $z$ is any point in $D$ (5) holds for $\zeta=\mathrm{Rz}$ where $-\delta<R<\delta$. Letting $R \rightarrow-\delta$ we obtain (12). Equation (12) also holds when $\delta=0$. If we let 


$$
F(z)=\frac{z f^{\prime}(z)}{f(z)-f(-\delta z)}=\sum_{n=0}^{\infty} c_{n} z^{n},
$$

then $\left|c_{n}\right| \leqq 2 \operatorname{Re} c_{0}=2(1+\delta)^{-1} ;(\operatorname{see}($ Robertson (1945))). We have the following theorem:

THEOREM 3. If $w=f(z) \in S_{\delta}$, then

$$
\left|a_{n}\right| \leqq \prod_{k=1}^{n-1} \frac{k(1+\delta)+1-(-\delta)^{k}}{k(1+\delta)+1+(-\delta)^{k+1}},
$$

equality holding in the cases $\delta=0$ and $\delta=1$.

Proof. Equation (13) gives the following relationship between the coefficients of $w=f(z)$ and $F(z)$

$$
\left(n-c_{0}\left(1-(-\delta)^{n} a_{n}=\sum_{k=1}^{n-1} c_{k} a_{n-k}\left(1-(-\delta)^{n-k}\right),\right.\right.
$$

equality holding in the cases $\delta=0$ and $\delta=1$.

Let

$$
\begin{aligned}
P_{k} & =k+\sum_{j=0}^{k-1} \rho^{j}, \\
Q_{k} & =\sum_{j=0}^{k-1}(k-j) \rho^{j}, \quad k=1,2, \cdots .
\end{aligned}
$$

Let $S_{1}=(1-\rho)$, and define $S_{n}$ recursively by

$$
S_{n}=S_{n-1}+\frac{1-\rho^{n}}{(1-\rho)^{n-1}} \prod_{k=1}^{n-1} \frac{P_{k}}{Q_{n}}, n=2,3, \cdots .
$$

Set

$$
T_{n}=\frac{1}{(1-\rho)^{-1}} \frac{Q_{n}}{2} \prod_{k=1}^{n} \frac{P_{k}}{Q_{k}} .
$$

We will prove the following identity;

$$
S_{n}=T_{n}, n=1,2 \cdots .
$$

Assuming (16) for $n=m-1$, we have

$$
\begin{aligned}
S_{m} & =\frac{1}{(1-\rho)^{m-3}} \frac{Q_{m-1}}{2} \prod_{k=1}^{m-1} \frac{P_{k}}{Q_{k}}+\frac{1-\rho^{m}}{(1-\rho)^{m-1}} \prod_{k=1}^{m-1} P_{k} / Q_{k} \\
& =T_{m}\left[(1-\rho) Q_{m-1} / P_{m}+2 \frac{\left(1-\rho^{m}\right)}{(1-\rho)} P_{m}\right]=T_{m} .
\end{aligned}
$$

Since (16) is easily verified for $n=1,(16)$ follows. 
Let $\rho=-\delta$. We will prove that

$$
\left|a_{n}\right| \leqq \frac{1}{(1-\rho)^{n-1}} \prod_{k=1}^{n-1} \frac{P_{k}}{Q_{k}}, n=2,3, \cdots
$$

These estimates are obtained from (15) by induction when we use $c_{0}=(1+\delta)^{-1}$, the bounds $\left|c_{n}\right| \leqq 2(1+\delta)^{-1}$ and the estimates for $\left|a_{n-1}\right|,\left|a_{n-2}\right|$, etc.

For $n=2,(15)$ gives $a_{2}=c_{1}(1+\delta)\left(2-c_{0}\left(1-\delta^{2}\right)\right)^{-1}$. Hence $\left|a_{2}\right| \leqq$ $2(1+\delta)^{-1}$, which gives (14) for $n=2$. Assume now that (15) holds for $k \leqq n-1$. Then (15) gives

$$
a_{n}=\frac{1}{n-c_{0}\left(1-\rho^{n}\right)} \sum_{k=1}^{n-1} c_{k} a_{n-k}\left(1-\rho^{n-k}\right)
$$

Hence,

$$
\left|a_{n}\right| \leqq \frac{2 \sum_{k=1}^{n-1}\left|a_{k}\right|\left(1-\rho^{k}\right)}{n(1-\rho)-\left(1-\rho^{n}\right)}=\frac{2 \sum_{k=1}^{n-1} a_{k} \mid\left(1-\rho^{k}\right)}{(1-\rho)^{2} Q_{n-1}} .
$$

By the induction hypothesis and (16) we have

$$
\left|a_{n}\right| \leqq \frac{2 S_{n-1}}{(1-\rho)^{2} Q_{n-1}}=\frac{2 T_{n-1}}{(1-\rho)^{2} Q_{n-1}}=\frac{1}{(1-\rho)^{\alpha-1}} \prod_{k=1}^{n-1} P_{k} / Q_{k},
$$

and (17) is satisfied.

We now show that (17) is equivalent to (14). Note that

$$
P_{k}=k+\left(1-\rho^{k}\right) /(1-\rho) \text {. }
$$

and

$$
(1-\rho) Q_{k}=k-\rho\left(1-\rho^{k}\right) /(1-\rho)
$$

Hence,

$$
\frac{P_{k}}{1(-\rho) Q_{k}}=\left[k(1-\rho)+1-\rho^{k}\right]\left[k(1-\rho)+\rho+\rho^{k+1}\right]^{-1} .
$$

If we combine (17) and (18) we obtain (14).

We conclude with an example. It has been suggested that if $w=f(z) \in S_{\delta}$, then there may exist some $\beta>0$, depending on $\delta$, such that

$$
\inf _{z \in D} \operatorname{Re} \frac{z f^{\prime}(z)}{f(z)}>\beta \text {. }
$$

The functions

$$
f_{\beta}(z)=\frac{1}{2 \beta}\left[1-\left(\frac{1+z}{1-z}\right)^{\beta}\right]
$$


serve as a counterexample in the following sense. As $\beta$ varies in the interval $[0,1]$, $f_{\beta}(z)$ has an index that decreases with respect to $\beta$ in the interval $[0,1]$. Furthermore

$$
\inf _{z \in D} \operatorname{Re} \frac{z f_{\beta}^{\prime}(z)}{f_{\beta}(z)}=0
$$

for all $\beta, 1 \leqq \beta \leqq 2$.

\section{References}

Z. Nehari, Conformal Mapping (1952), (McGraw-Hill, New York, 1952, MR 13, 640.)

M. S. Robertson (1945), 'Star center points of multivalent functions', Duke Math. J. 12, 669-684; MR 7-379.

T. J. Suffridge (1940), 'Some remarks on convex maps of the unit disk', Duke Math.J. 37, 775-777; MR 42-852.

E. Strohhäcker (1933), 'Beitrage zur Theorie der Schlichten Funcktionen', Math. Z. 37, 336-380; Zbl. 7-214.

Temple University, Philadelphia, Penna., U.S.A.

The Institute for Advenced Study, Princeton, N. J. U.S.A. 